\title{
COMPENSATING THE FREQUENCY DEADBAND OF THE APS REAL-TIME AND DC TRANSVERSE ORBIT CORRECTION SYSTEMS*
}

\author{
C. Schwartz ${ }^{\dagger}$ and L. Emery \\ Advanced Photon Source, Argonne National Laboratory, Argonne, IL
}

\begin{abstract}
Currently, two parallel feedback systems correct the transverse Advanced Photon Source (APS) orbit in both planes: a workstation-based slow (DC) system and a realtime system (RTFS) that samples at $1.6 \mathrm{kHz}$. A partial solution to preventing feedback-system crosstalk has been to bandlimit the RTFS from below, but this fix has borne a system deadband in which little orbit correction is achieved. We present a matrix time-domain compensation algorithm that greatly reduces the system deadband, as well as operational data.
\end{abstract}

\section{PROBLEM STATEMENT}

The Advanced Photon Source (APS) real-time [1] and DC [2] orbit correction systems currently run in parallel, and both attempt global correction. The first uses 38 high-bandwidth correctors and about 160 beam-position moniters (BPMs) in each plane and operates at a $650 \mu \mathrm{s}$ sampling interval. The workstation-based DC system uses 80 correctors and all available BPMs $(>300)$ and updates its correction at intervals of between 2.5 and 4 seconds. The real-time system provides wide band correction, but cannot correct as many spatial modes as the DC system (because of its relatively small number of correctors); it also cannot make use of BPM "despiking" [2], in which spurious BPM-signal offsets are ignored in feedback. In short, (the characteristics of) both systems are needed. However, when both systems are engaged simultaneously, they both attempt correction in a frequency band shared by their respective closed-loop bandwidths. The result is that the systems couple to, or "fight" each other, leading to an undesireable closed-loop orbit in the overlap band.

Until recently, this problem was partially solved by the following frequency-domain, scalar fix. The real-time system's bandwidth is cut off from below via a high-pass filter, such that its frequency band of correction does not overlap with that of the DC system. However, this is still only a partial solution: if we desire no effective overlap, we are left with a frequency deadband in which the beam orbit motion is not corrected at all. On the other hand, if we set the real-time system's low-frequency cutoff so that it is equal to the DC system's closed-loop bandwidth, there is still some fighting in the overlap band; certain modes are partially "corrected" by each system, and the composite orbit is worsened. This overlap/deadband problem has

\footnotetext{
* Work supported by U.S. Department of Energy, Office of Basic Energy Sciences, under Contract No. W-31-109-ENG-38.

$\dagger$ csch@aps.anl.gov
}

recently come to the fore, as many APS users move their insertion-device (ID) gaps at rates that happen to fall in the band in question. These effective step changes to the beam orbit are globally felt, and are ill corrected when both systems are in operation; the orbit oscillates with a period of nearly a minute, and it takes over one minute to decay to zero.

In this paper, we report on a new solution to this problem. The solution takes into account the problem's inherent spatiality, which is the source of the systems' undesired coupling. We first present a theoretical framework for the orbit feedback problem (Section 2). It is from this formulation that the solution immediately suggests itself. Section 3 presents the results of our implementation.

\section{THEORY}

\subsection{A Single-System Transfer Function Matrix}

Following the notation of [3], we denote the $M$-tuple vector of orbit readings (on the BPMs) at discrete-time in$\operatorname{dex} k$ as $\mathbf{x}[k]$. In $z$-transform domain [4], this vector is denoted as $\mathbf{x}(z)$. Assuming for now that only one feedback system is in operation (either DC or real-time), then orbit motion arises in two sources: ambient noise and magnetinduced correction via the feedback system. We denote these two contributions in $z$-space respectively as $\mathbf{w}(z)$ and $\mathbf{u}(z)$. Thus, we have $\mathbf{x}(z)=\mathbf{u}(z)+\mathbf{w}(z)$.

The feedback system correction $\mathbf{u}(z)$ is obtained by resolving the beam's orbit $\mathbf{x}(z)$ onto $N$ correctors via an inverse response matrix (viz., the response matrix psuedo inverse). That is, the ( $N$-tuple) so-called "corrector-error" vector is given by

$$
\mathbf{c}_{\mathrm{E}}(z)=\mathbf{R}^{+}[\mathbf{s}-\mathbf{x}(z)],
$$

where $\mathbf{s}$ is the (constant) BPM set-point vector. This corrector error is fed to a regulator (filter) from which a desired current signal is fed to the corrector power supplies as a $(N \times 1)$ command vector $\mathbf{c}[k]$. The effect of the corrector's resulting magnetic field on the beam is also bandlimited (i.e., not instantaneous) via eddy currents and zero-mode beam dynamics. To first order (for small signals), the totality of these dynamic effects can be represented by a linear $z$-domain transfer function (in general, unique for each corrector). Indeed, in the case of DC feedback, all but the regulator dynamics are negligible, and these are equivalent for each corrector. Thus, for the DC case, the corrector-error to feedback-system correction (matrix) transfer function is 
given by

$$
\mathbf{u}(z)=\frac{G}{z-1} \mathbf{R c}_{\mathrm{E}}(z),
$$

where $\mathbf{R}$ is the response matrix, and $G$ is the system gain. An equivalent time-domain version of this equation is

$$
\mathbf{c}[k]=\mathbf{c}[k-1]+G \mathbf{c}_{\mathrm{E}}[k-1]
$$

since $\mathbf{R}^{+} \mathbf{R}=\mathbf{I}$ [3]. If, unlike the DC-system case, the dynamics of the correctors are different, we have

$$
\mathbf{u}(z)=\mathbf{R H}(z) \mathbf{c}_{\mathrm{E}}(z)
$$

where $\mathbf{H}(z)$ is a diagonal matrix (since each corrector acts independently of the others) of scalar transfer functions.

Combining the above equations, we obtain the following key input-output relationship:

$$
\mathbf{x}(z)=\mathbf{G}(z)[\mathbf{w}(z)+\mathbf{Q}(z) \mathbf{s}]
$$

where $\mathbf{G}(z):=\left[\mathbf{I}+\mathbf{R H}(z) \mathbf{R}^{+}\right]^{-1}$ is referred to as the transfer-function matrix (TFM), and $\mathbf{Q}(z):=$ $\mathbf{G}(z) \mathbf{R H}(z) \mathbf{R}^{+}$. Equation (5) governs the closed-loop frequency-domain behavior of the orbit, given the ambient noise, the BPM set points, the response matrix, and the linearized system dynamics. Setting $z=e^{j \omega}$, it is possible to compute the feedback system's efficacy in rejecting noise at frequency $\omega$. Another application of the TFM is given below in the Appendix (Section 4).

\subsection{Two-System Parallel Orbit Feedback}

In the case of two parallel feedback systems, the vector of orbit readings $\mathbf{x}(z)$ is comprised of noise and the orbit corrections of the two systems; thus

$$
\mathbf{x}(z)=\mathbf{u}_{1}(z)+\mathbf{u}_{2}(z)+\mathbf{w}(z)
$$

To precisely characterize the deadband/overlap phenomenon, we extend the TFM formulation to two-system parallel orbit feedback.

A subtle formulation problem arises from the fact that each of the two feedback systems uses a particular set of BPMs to compute its corrector-error vector, and not all of these BPMs are shared between the two systems. To completely characterize the closed-loops' orbit, we must account for all of the BPMs used (i.e., the union of the two sets). Thus, each feedback system affects the same $M$ BPMs in our model, but uses only $M_{l} \leq M, l=1,2$ of them to compute the corrector error. Another way of saying this is that, although $\mathbf{x}(z)$ and $\mathbf{u}_{l}(z), l=1,2$ are $M \times 1$ vectors, each feedback system does not in general use all $M$ elements to compute the corrector error [cf. (1)]; instead system $l$ uses $M_{l}$ elements. Mathematically this is represented as

$$
\mathbf{u}_{l}(z)=\mathbf{R}_{l} \mathbf{H}_{l}(z) \mathbf{R}_{l}^{+} \mathbf{L}_{l}\left[\mathbf{s}_{l}-\mathbf{x}(z)\right], \quad l=1,2 .
$$

The matrix product $\mathbf{R}_{l} \mathbf{H}_{l}(z) \mathbf{R}_{l}^{+}$is of dimension $M \times M_{l}$, and the conforming matrix $\mathbf{L}_{l}\left(M_{l} \times M\right)$ simply "zeros out" those elements of $\mathbf{x}(z)$ not found in the inverse response matrix $\mathbf{R}_{l}^{+}$. The $l$ th system's TFM is $\mathbf{G}_{l}(z)=$ $\left[\mathbf{I}+\mathbf{R}_{l} \mathbf{H}_{l}(z) \mathbf{R}_{l}^{+} \mathbf{L}_{l}\right]^{-1}$.

A closed-form solution to Eq. (6) is obtained by first solving the coupled equations

$$
\begin{aligned}
& \mathbf{u}_{1}(z)=\mathbf{G}_{1}(z) \mathbf{Q}_{1}(z)\left[\mathbf{s}_{1}-\mathbf{w}(z)-\mathbf{u}_{2}(z)\right] \\
& \mathbf{u}_{2}(z)=\mathbf{G}_{2}(z) \mathbf{Q}_{2}(z)\left[\mathbf{s}_{2}-\mathbf{w}(z)-\mathbf{u}_{1}(z)\right]
\end{aligned}
$$

with $\mathbf{Q}_{l}=\mathbf{R}_{l} \mathbf{H}_{l}(z) \mathbf{R}_{l}^{+} \mathbf{L}_{l}, l=1,2$. (As stated, the solution of Eq. (8) requires $K$ inversions of a $2 M \times 2 M$ matrix, where $K$ is the number of frequency samples. However, using Schur complements, one can simplify the equations such that $2 K$ inversions of a $M \times M$ matrix are needed [5].)

\subsection{Overlap Compensation}

A solution to the coupled system, i.e., deadband, problem arises quite naturally from Eq. (8). If we set $\mathbf{s}_{2}$ equal to $\mathbf{u}_{1}(z)$, then system two is unaffected by system one. That is, the anticipated orbit contribution of system one is sent to system two and effectively subtracted from the latter's set-point vector. Note that we cannot use two feedforwards to mutually decouple the two systems, because then both systems would attempt to control the orbit, leading to undesirable (unobservable) dynamics.

We have implemented the above system-to-system "feedforward" as a solution to the deadband problem. We have chosen to feed from the DC system to the RTFS; thus, system indices 1-2 of Eq. (8) refer to the DC and RTFS respectively. This choice requires the DC system to modify the RTFS-BPM setpoints with reasonable speed. The opposite choice (i.e., feeding from the RTFS to the DC system) was ruled out because, among other reasons, the DC system's (computer network) timing jitter would be sensitive to the very fast RTFS update rate. In short, our compensation amounts to adjusting the real-time BPM set points $\mathbf{s}_{2}$ with the expected orbit contribution of the DC system, $\mathbf{u}_{1}[k]$, where $\mathbf{u}_{1}[k]$ is according to Eq. (2), and discretetime index $k$ increments with the DC-system update time. In practical terms, the DC system at each iteration must write the result of Eq. (3), viz., the DC correctors' desired current, and also write new RTFS BPM setpoints $\mathbf{s}_{2}$ according to $\mathbf{s}_{2}=\mathbf{L}_{2} \mathbf{R}_{1} \mathbf{c}_{1}[k]$.

\section{RESULTS}

\subsection{Computational Results}

The coupled-loops system behavior governed by Eqs. (6) and (8) was computed, both with and without feedforward compensation, using the horizontal plane as an example. Given the high speed of the real-time system's response, it is sufficient to model that system's dynamics as only a high-pass filter, with a cutoff of $0.5 \mathrm{~Hz}$ and a gain of 25. The DC system's (empirically) optimal parameters are with a gain $G=0.4$, and a $T=2.5$-second update interval. (Without compensation, it is impossible to run with 
these parameters because of the system cross-coupling; in absence of feedforward, we have used $G=0.15, T=4.0$ in operations.) As shown in Fig. 1, the system's computed uncompensated response to white noise on corrector S20A:H1, as seen on BPM S20A:P1 (0.05 betatron radianphase advance), exhibits a deadband centered at about 0.02 $\mathrm{Hz}$. The figure shows that, for this particular experiment, a two-fold improvement at that frequency is achieved with the feedforward compensation.

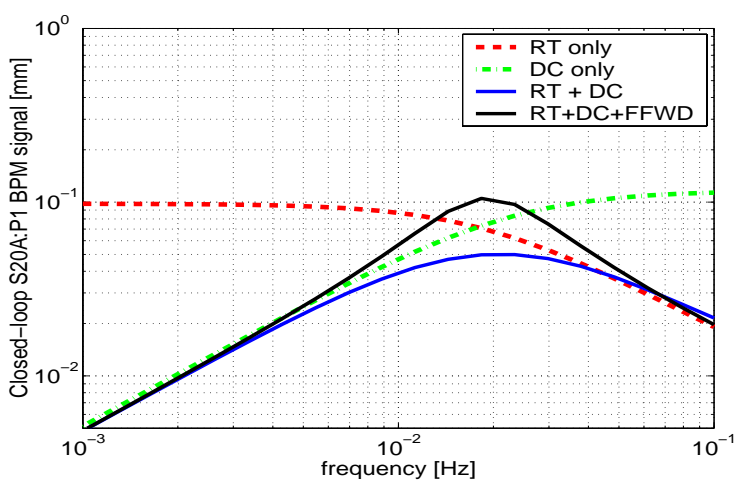

Figure 1: BPM S20A:P1 computed closed-loop responses to white noise on a nearby corrector.

\subsection{Empirical Results}

We present before-and-after results for the horizontal plane case in Fig. 2. Evidently, the DC-system-only response is satisfactory in the steady state but suffers from high-frequency noise; the reverse is true for RTFS-only response. Both systems operating in parallel induce a $\sim 0.02-$ $\mathrm{Hz}$ oscillation. The compensation removes the oscillation, however, some discontinuous beam motion arises as a result of stepping the real-time BPM set points at the DCsystem update rate; this is the price paid for improved lower-frequency response. Fig. 3 shows before-and-after results for ID gap movement. A further operational consequence of the algorithm is that we can ramp a circularly polarized undulator ID device four times faster and with a three-fold decrease in peak orbit distortion than before. As of late May 2001, the compensation algorithm was installed for both planes.

\section{APPENDIX}

We mention here parenthetically that the TFM can be used to compute the feedback system spatial "noise floor," i.e., the maximum achievable correction of betatron modes. The method is illustrated for a DC-system only (but applies to parallel systems as well). Note first that $\mathbf{R}^{+}=$ $\left(\mathbf{R}^{\prime} \mathbf{R}\right)^{-1} \mathbf{R}^{\prime}$, with ' denoting transpose; therefore, $\mathbf{R}^{+} \mathbf{R}=$ I. A further important fact [3] is that $\mathbf{G}(z)=\mathbf{I}-$ $\mathbf{R H}(z)\left[\mathbf{I}+\mathbf{R}^{+} \mathbf{R H}(z)\right]^{-1} \mathbf{R}^{+}$, with $\mathbf{H}(z)=G / z-1$. At DC $z \equiv 1$, hence, $\mathbf{x}(1)=\left[\mathbf{I}-\mathbf{R R}^{+}\right] \mathbf{w}(1)$. This last equation quantifies the spatial noise floor: since (in the typ-

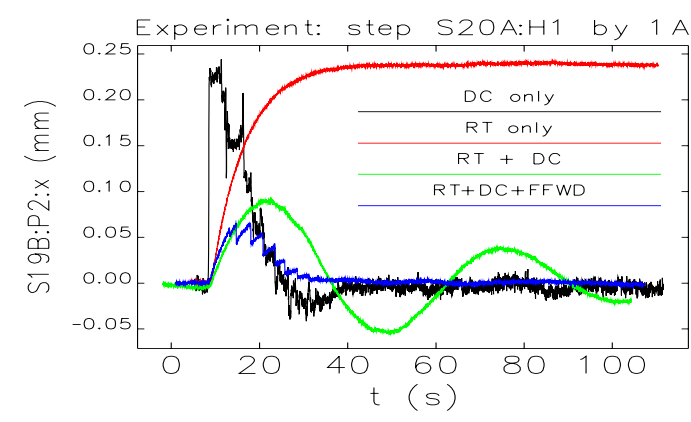

Figure 2: Response to step on nearby corrector.

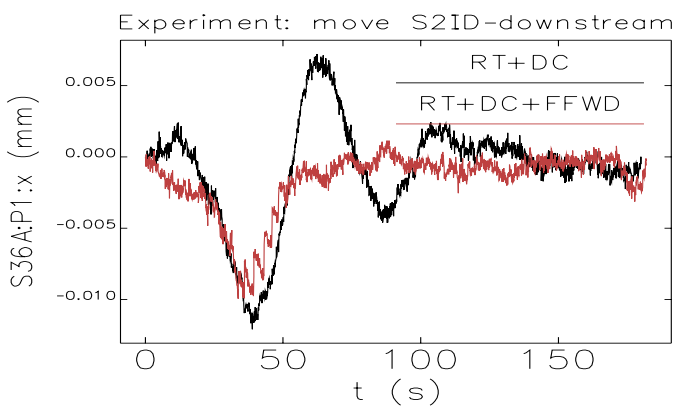

Figure 3: Compensated and uncompensated response to ID-gap movement.

ical case) $\mathbf{R} \mathbf{R}^{+} \neq \mathbf{I}$, there will always be residual orbit motion irrespective of the value of the integrator gain $G$, compare [6].

\section{ACKNOWLEDGMENTS}

The authors wish to thank J. Carwardine, O. Singh, G. Decker, and F. Lenkczus of APS for helpful technical insight. The latter individual also assisted in the algorithm's implementation.

\section{REFERENCES}

[1] J.A. Carwardine and F.R. Lenkszus, "Real-Time Orbit Feedback at the APS," Proc. Eighth Beam Instrumentation Workshop (1998).

[2] L. Emery and M. Borland, "Advances in Orbit Drift Correction in the Advanced Photon Source Storage Ring," Proc. 1997 Particle Accelerator Conference (1997).

[3] C. Schwartz, "Modeling Transverse Orbit Feedback Control," LS-289, September 2000, available at http://www.aps.anl.gov/techpub/lsnotes/ls289.pdf

[4] G. F. Franklin, J. D. Powell, and A. Emami-Naeini, Feedback Control of Dynamic Systems, 3 Ed. (1994).

[5] "A Matlab Toolkit for Orbit Feedback Computations," C. Schwartz, In preparation.

[6] Y. Chung, "A Unified Approach to Global and Local Beam Position Feedback," Proc. 1994 European Particle Accelerator Conference (1994). 